\section{The truth about physics}

\section{J. L. Heilbron}

The Creative Moment: How Science Made Itself Alien to Modern Culture. By Joseph Schwartz. Jonathan Cape/HarperCollins. Pp. 252. £16.99, \$25.

MANY will share Schwartz's indignation at our greedy, ignorant despoliation of our planet. Fewer, perhaps, will accept his diagnosis that the fatal flaw in our civilization is the refusal of almost all of us to learn enough about our physical world to understand the environmental consequences of our greed or the beauty of the science our acquisitiveness exploits. Fewer still will follow Schwartz in identifying misunderstanding of the theory of relativity as an important cause of the decline of the West.

The route by which he arrives at this last insight is as arresting as the insight itself. According to Schwartz, Galileo and Newton started the trouble by couching physics in terms of mathematics. They did so from no necessity immanent in the subject matter but to ensure the survival of physics in a hostile political and religious environment. "If physics were to survive in a socially divided Europe, it had to be well hidden". The message of Galileo was "keep it quiet, keep it obscure, and keep it mathematical". Newton's Principia, according to Schwartz, can easily be decoded to reveal its physical content apart from its mathematics. The example he offers, which reasons from "the observed acceleration of the planet Mercury in its orbit", is not reassuring. Mercury's acceleration is not an observation but an imputation, authorized and confirmed by the mathematics of the theory of gravity.

The culmination of nineteenth-century physics, according to Schwartz, is the theory of relativity. Once again, mathematics veiled physics from the people. Once again, Schwartz guts the physics to make his point. The entire physical content of the special theory of relativity, he says, is that all physical interactions require time. But as the case of the propagation of sound waves in air suggests, it is not possible to deduce from the principle of action in time that there exists a maximum permissible velocity that has the same value for everyone in the Universe. The deduction requires additional postulates, and the demonstration of their counter-intuitive consequences requires mathematics.

Just as physicists of Newton's time missed the chance to make their subject popular, so in Schwartz's rendition of history their descendants in the 1920 s, failing to take advantage of the popular enthusiasm for Einstein, managed to make the beautiful and transparent theory of relativity a byword for the difficult and obscure. In this we may gue. Schwartz is the author of a book intended to make Einstein's theories easy. He must often have encountered the strong resistance of humankind to mathematical reasoning, even when covert, and people's general distaste for mathematicians. Benjamin Franklin's tribute to his friend Thomas Godfrey may stand for many expressions of this antipathy. Godfrey was not "a pleasing companion," Franklin wrote, "as like most mathematicians I have met with, he expected unusual precision in everything said, or was forever denying or distinguishing upon trifles to the disturbance of all conversation." Because of its mathematics, physics will not appeal to all tastes. It can be popularized qualitatively and usefully; but the result is not physics.

Much of the rest of Schwartz's arguhear the cry of the proselytizing pedago(1711) shows various ment concerns the separation of the cultures of production and consumption. With the physicists' conspicuous failure to popularize their subject, consumers lost track of the physical consequences of consumerism. Physics itself split into a theoretical part, increasingly removed from nature, and an experimental part, closer to real things and consequently further from the pinnacle of arrogance where the élite of the élite, the theorists, prance without advancing. The failure of the physicists during the 1920 s to understand quantum mechanics coincided with the failure of popular political revolutions in Central Europe and also with the failure of new art-forms, such as cubism and music on the twelve-tone scale, to establish themselves. The physicists did succeed in one thing, however: they attracted the support of the Rockefeller Foundation. That was a mistake. Physicists supported by the foundation erred in thinking that they retained control of their work. "The political turning point had passed. Alamogordo was now very near."

Having come so far, Schwartz attacks a menagerie of bêtes noires: General Groves and the Manhattan Project, for duping physicists to make a bomb to bully the Soviet Union; the Rockefeller Foundation again, for converting molecular biology into a mechanistic science;

\title{
Time on one's hands
}

During the sixteenth and seventeenth centuries, diptych sundials became popular devices for telling the time. Most diptych dials have a string gnomon (shadow. caster) which stretches at an angle (determined by the latitude of the observer) between the two tablets. Magnetic compasses for correctly orientating the dials were probably first added before the fifteenth century. This Illustration from a work by Johannes Gaupp styles of compass needles and bowls; it is reproduced in Ivory Diptych Sundlals, 1570-1750, a beautifully illustrated catalogue of Harvard University's collection of 82 of these timepieces. The collection, which is one of the largest in the world, consists mostly of designs from Nuremberg (the first clty in Europe to have an organized trade of compass-makers), Paris and Dieppe. The book is distributed by Harvard University Press, £39.95.

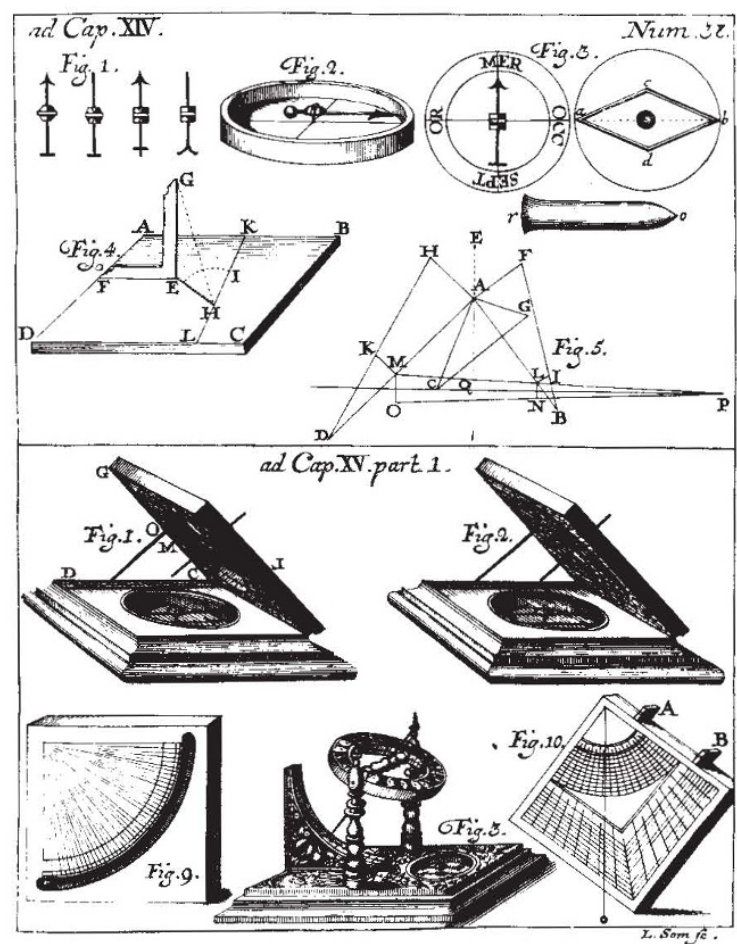

